\title{
FAST, MEMS-BASED, PHASE-SHIFTING INTERFEROMETER
}

\author{
Hyuck Choo ${ }^{1}$, Rishi Kant ${ }^{2}$, David Garmire ${ }^{1}$, James Demmel ${ }^{1}$, and Richard S. Muller ${ }^{1}$ \\ ${ }^{I}$ Berkeley Sensor \& Actuator Center, University of California, Berkeley, CA USA \\ ${ }^{2}$ Department of Electrical Engineering, Stanford University, Stanford, CA USA
}

\begin{abstract}
We demonstrate a MEMS-based, phase-shifting interferometer (MBPSI) that is much faster than conventional phase-shifting interferometers (PSI). For phase shifting, our system employs a comb-driven vertically resonating micromachined mirror illuminated by synchronized laser pulses $(\lambda=660 \mathrm{~nm})$. Our MBPSI employs a four-frame phase-shifting technique (four CMOS-imager frames per one profile measurement), at a rate of 23 profile measurements-per-second $(23 \mathrm{~Hz}, 43.5 \mathrm{msec}$ per measurement). At this rate, the MBPSI can continuously capture more than 500 profile measurements of a transient phenomenon over 21.7 seconds. The MBPSI in Twyman-Green configuration has accurately tracked in real time the fast-changing, transient motion of a PZT actuator, within $\pm \lambda / 110( \pm 6 \mathrm{~nm})$.
\end{abstract}

\section{INTRODUCTION}

PSIs are desired for precision measurements of moving objects, and increasing the PSI data-collection rate makes it possible to observe faster motions. Our MBPSI system can capture 500 or more profile measurements of a transient phenomenon continuously at a rate of $23 \mathrm{~Hz}$. Besides sizably expanding the range of PSI applications (for example, to transient optical phenomena such as chemical diffusion, crystal growth, and measurements of rapidly varying object temperatures [1-2]), the MBPSI's rapid measurement rate reduces the data noisiness caused by low-frequency vibrations. Batch-fabricated, MEMS phaseshifting components are not only faster, but also much more costeffective to fabricate and operate than are the piezoelectric actuators that are now typical in PSI systems.

\section{FAST PHASE-SHIFTING METHOD}

Our phase-shifting technique (illustrated in Figure 1) is, in principle, similar to that used to obtain a stroboscopic movie of a periodically moving object.

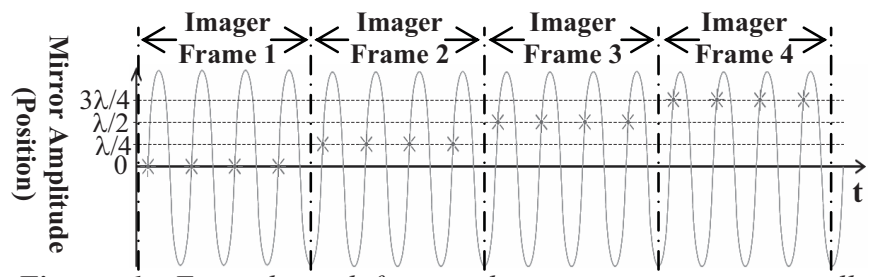

Figure 1. Fast phase-shifting technique using our vertically resonating micromirror and a pulsed laser diode: '米' indicates when the laser pulse is flashed. In this example, there are four phase steps; each frame of the CMOS imager integrates images generated by four laser pulses flashed for each phase step.

In the MBPSI, we obtain stable interference patterns using a periodically moving mirror and synchronized laser pulses. The interference patterns corresponding to $0, \lambda / 4, \lambda / 2$, and $3 \lambda / 4$ phase shifts are captured in the CMOS-imager frames 1, 2, 3, and 4 , respectively [3]. In each frame, while driving a MEMS mirror at its resonant frequency, we pulse the laser diode when the mirror is displaced by the desired fraction of the illuminating wavelength from its initial position. Because the integrating-bucket technique for CMOS imagers requires that the movement of the phase shifter be linear [3], we use the linear region of the mirror's resonant motion, which is in the beginning of each period, as shown in Figures 1 and 5. When using this technique, the maximum profilemeasurement rate is equal to the CMOS-imager frame rate (fps) divided by the number of phase steps required.

\section{MEMS PHASE-SHIFTING MIRROR: FABRICATION AND CHARACTERIZATION}

The phase-shifting mirror measures 5 by 5 by $0.05 \mathrm{~mm}$ and is micromachined using a fabrication method that we have described earlier [4] and applied to produce high-precision torsional microscanners (Figure 2).

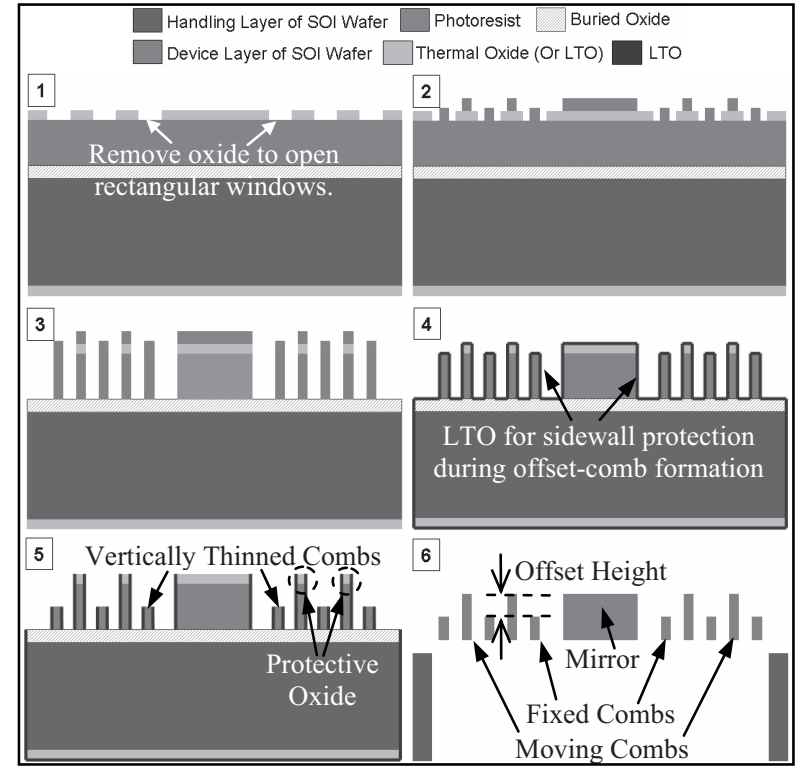

Figure 2. Fabrication Process: The left column shows top views while the right column shows cross-sectional views along the dotted lines in our CMOS-compatible mirror-fabrication process. 1. Grow 0.5- $\mu$ m thermal or low-temperature oxide (LTO). Using the photolithography mask \#1, pattern and remove the thermal oxide selectively where fixed combs will be later fabricated; 2. Using mask \#2, create patterns of micromirror including moving and fixed combs, flexures, and mirrors; 3. Use deep-reactive-ion-etch (DRIE) to define the micromirror in the device layer; 4. Remove the photoresist layer and deposit a very thin layer $(\sim 0.2 \mu \mathrm{m})$ of LTO; 5. Use timed-anisotropic-plasma etch to remove 0.2- $\mu$ m thick LTO from the top-facing surfaces. Then use timed-anisotropic or isotropic silicon-etch to create a set of vertically thinned combs; and 6. Using mask \#3, pattern and open the backside of the micromirror. Release the devices in HF and perform critical-point drying.

A new feature of our mirror design for the MBPSI is the incorporation of stress-relieving beams which improve the flatness of the mirror (Figures 3 and 4). We have measured the surface profile of a representative mirror (of more than 100 that we have produced) using a WYKO NT3300 (Figure 3) and determined that its radius of curvature exceeds $20 \mathrm{~m}$, and its surface-roughness values do not exceed $20 \mathrm{~nm}$. In order to time the laser pulses precisely, the mirror resonance has been analyzed using a piezobased, calibrated stroboscopic interferometer (Figure 5) [5]. The 
measured resonant frequency and mechanical quality factor of the mirror are $3.55 \mathrm{kHz}$ and 63 , respectively. When the mirror achieves a resonant amplitude of $1.5 \mu \mathrm{m}$ (actuation voltage $\left.=18 \mathrm{~V}_{\text {ac } \_ \text {-to- } p}\right)$, the pulses $(1-\mu$ sec duration) for $0, \lambda / 4, \lambda / 2$, and $3 \lambda / 4(\lambda=660 \mathrm{~nm})$ occur at intervals of $0,10,20$, and $30 \mu \mathrm{sec}$ (Figure 5). Dynamic deformation of the mirror, between the center and the four corners of the mirror, within this linear region of operation, is less than $6 \mathrm{~nm}(\lambda / 110)$ (Figure 5).

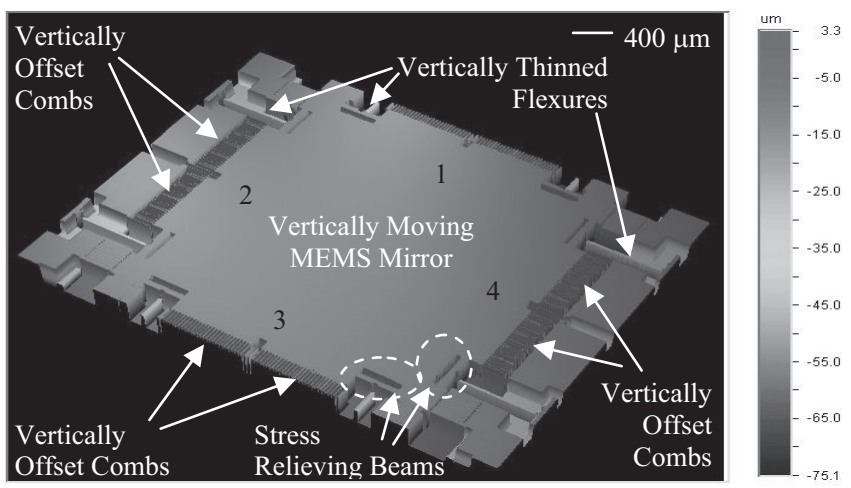

Figure 3. WYKO profile measurement of fabricated MEMS mirror

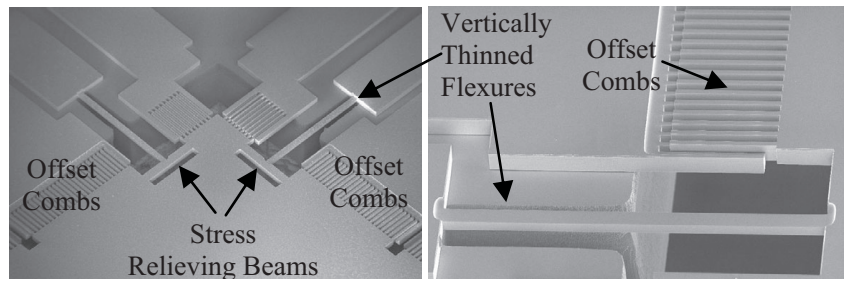

Figure 4. SEM images showing the key sections of the phaseshifting MEMS mirror
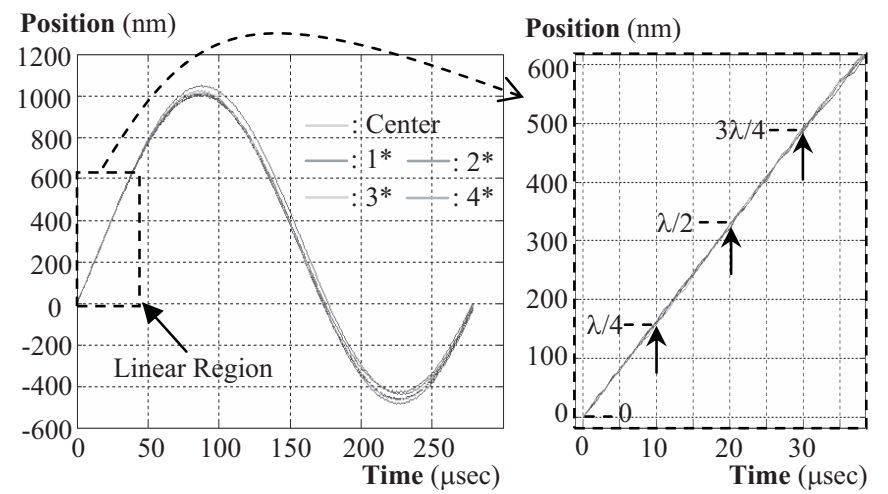

Figure 5 Resonant-motion analysis for our phase-shifting MEMS mirror using calibrated laboratory Stroboscopic Interferometer Left: One full period of resonant motion, with the linear region indicated by the red rectangle, Right: Linear region used for phaseshifting (peak-to-peak deviation in position: < $6 \mathrm{~nm})(* 1-4$ : Please refer to Figure 3.)

\section{OPTICAL MEASUREMENT RESULTS OF MBPSI}

The MBPSI in Twyman-Green configuration (Figure 6) has tracked in real time the fast-changing, transient motion of a PZT actuator [6]. The actuator was stepped at intervals of $100 \mathrm{~nm}$ every $0.5 \mathrm{sec}$ over a 6.478 -sec period (150 profile measurements, Figure 7). The maximum frame rate of our CMOS-imager is $100 \mathrm{fps}$. We ran the imager at a conservative $92 \mathrm{fps}$, reflecting a $23 \mathrm{~Hz}$ profilemeasurement rate. The transient measurement was precise to within $\pm 6 \mathrm{~nm}( \pm \lambda / 110)$, lower than the $10 \mathrm{~nm}$-accuracy limit of the PZT-actuator movement (from the readings of the PZT's built-in feedback position sensor), as shown in Figure 7.

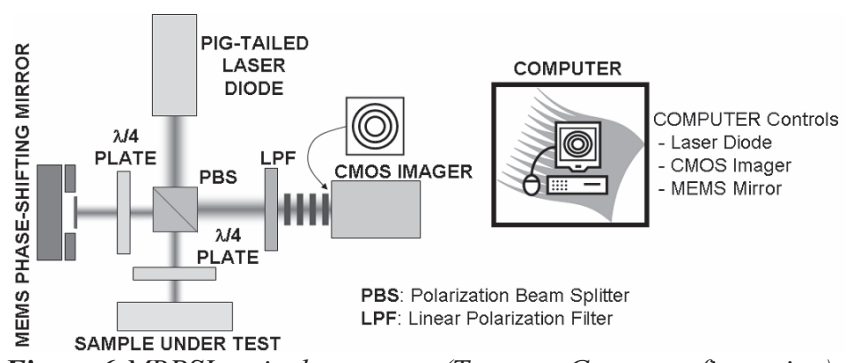

Figure 6 MBPSI optical test setup (Twyman-Green configuration)

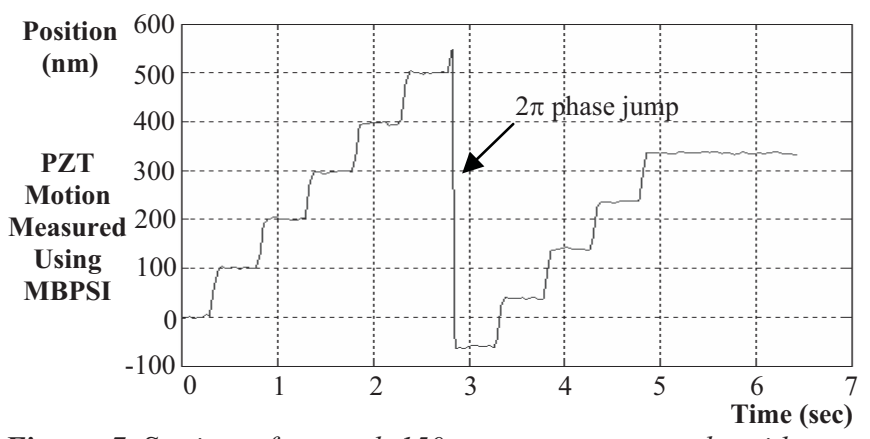

Figure 7 Section of a total 150 measurements made with our MBPSI system showing a PZT actuator moving at a step of $100 \mathrm{~nm}$ every 0.5 seconds during the 6.478-second period (PZT movement resolution: $< \pm 10 \mathrm{~nm}$ from the readings of the PZT built-in feedback position sensor)

\section{CONCLUSIONS}

We have demonstrated a fast, accurate, MEMS-based PSI, which can continuously measure transient optical phenomena at $23 \mathrm{~Hz}$. We plan to report long-term repeatability, analysis for the effects of various error/noise sources, performance in the presence of mechanical vibrations, and new application(s) in our presentation.

\section{ACKNOWLEDGEMENT}

The authors thank Prof. J. Bokor of UC Berkeley and Prof. C. R. Pollock of Cornell University for helpful discussions on interferometry techniques and optical fiber properties. We also thank the National Science Foundation for generously funding our research (NSF EEC0318642 and CITRIS NSF TR22325).

\section{REFERENCES}

[1] K. Onuma, K. Tsukamoto, and S. Nakadate, "Application of real-time phase shift interferometer to the measurement of concentration field," J. of Crystal Growth, vol. 129, 1993, pp. 706-718

[2] P. V. Farrell, G. S. Springer, and C. M. Vest, "Heterodyne holographic interferometry- Concentration and temperature measurements in gas mixtures," Applied Optics, vol. 21, no. 9, May 1982, pp.1624-7, USA

[3]D. Malacara, Ed., "Optical Shop Testing," $2^{\text {nd }}$ Edition, Chap. 14, New York: Wiley, 1992, USA

[4] H. Choo, D. Garmire, J. Demmel, and R. S. Muller, "A Simple Process to Fabricate Self-Aligned, High-Performance Torsional Microscanners: Demonstrated Use in a Two-Dimensional Scanner," 2005 IEEE/LEOS International Conference on Optical MEMS and Their Applications, August 1-4, 2005, pp.21-22, Oulu, Finland

[5] M. R. Hart, R. A. Conant, K. Y. Lau, and R. S. Muller, "Stroboscopic Interferometer System for Dynamic MEMS Characterization," J. MEMS, vol. 9, no. 4, December 2000, pp. 409-418, USA

[6] PZT Actuator: PI P-753 LISA, PZT Controller: PI E-501 